\title{
Blood pressure goal attainment in multi-ethnic Asian patients with hypertension and dyslipidaemia in primary care
}

Kim Hwee $\underline{K o h}^{1,2}$, MMed, FCFPS, Chin Chin $\underline{G o h}^{3}$, BPharm, Soo Chye Paul Goh ${ }^{4}$, MMed, FCFPS, Yi Ling Eileen $\underline{K o h}{ }^{5}$, BSc, Ngiap Chuan $\underline{T a n}^{5}$, MMed, FCFPS

\begin{abstract}
INTRODUCTION Hypertension and dyslipidaemia are major risk factors for cardiovascular diseases and achieving treatment goals mitigates such risks. This study determined demographic and medication-related factors associated with blood pressure (BP) goal attainment in patients with concomitant hypertension and dyslipidaemia.

METHODS This paper is a sub-analysis of the Lipid HEALTH study, a questionnaire survey focusing on adult Asian patients with both dyslipidaemia and hypertension. An interviewer-administered questionnaire was used to obtain demographic and clinical information. Laboratory and prescription data was retrieved from electronic health records. BP goals were defined by international guidelines. Data was audited and analysed, and logistic regression analysis was used to identify factors determining BP goal attainment.

RESULTS Among the 851 included patients, $49.7 \%$ attained their BP goals. $37.0 \%$ were on monotherapy, $57.6 \%$ on $\geq 2$ BP-lowering medications, and $5.4 \%$ had no pharmacologic treatment. Among those on pharmacotherapy, $51.2 \%$ failed to achieve BP goals. Calcium channel blockers were the most frequently prescribed medications. Attainment of BP goals was significantly associated with: not having Type 2 diabetes mellitus (odds ratio [OR] 2.27, 95\% confidence interval [CI] 1.61-3.13); attaining low-density lipoprotein cholesterol goal (OR 2.02, 95\% Cl 1.45-2.81); being solely on dietary control (OR 2.19, 95\% Cl 1.09-4.39); and receiving monotherapy (OR 1.71, 95\% Cl 1.18-2.48).

CONCLUSION BP treatment goals were attained by half of the patients with dyslipidaemia and hypertension, and half of those on pharmacotherapy. Type 2 diabetes mellitus and low-density lipoprotein cholesterol control were significantly associated with BP goal attainment.
\end{abstract}

Keywords: blood pressure, dyslipidaemia, hypertension, treatment goal

\section{INTRODUCTION}

Hypertension and dyslipidaemia are highly prevalent cardiovascular risk factors, and the two conditions frequently coexist. ${ }^{(1)}$ In the United States, more than three-quarters of hypertensive patients have dyslipidaemia, with fewer than $20 \%$ of cases under control, according to the National Health and Nutrition Examination Surveys for the period 2005-2010. ${ }^{(1)}$

Hypertension and dyslipidaemia are both independently associated with coronary heart disease (CHD), and the presence of both conditions concomitantly presents an additive risk of cardiovascular events. ${ }^{(2,3)}$ The Seventh Report of the Joint National Committee on Prevention, Detection, Evaluation and Treatment of High Blood Pressure (JNC 7)(4) and the European Society of Hypertension-European Society of Cardiology guidelines for the management of arterial hypertension ${ }^{(5)}$ show that even small elevations above blood pressure (BP) goals increase the likelihood of developing a cardiovascular disease.

Treatment guidelines such as the JNC $7^{(4)}$ and the National Cholesterol Education Program Adult Treatment Panel III guidelines $^{(6)}$ have established optimal BP and low-density lipoprotein cholesterol (LDL-C) treatment goals, respectively, based on a patient's overall cardiovascular risk. These guidelines also recommend simultaneous management of multiple risk factors such as hypertension and dyslipidaemia. ${ }^{(4,6)}$ Treating BP to goals was associated with a reduction in residual CHD risk by $20 \%-25 \%$ in hypertensive patients, while treating associated dyslipidaemia in hypertensive patients reduced residual CHD risk by $35 \%-40 \% .{ }^{(1)}$ However, despite these established guidelines and the importance of lowering BP and lipid levels for the prevention of cardiovascular events, it is estimated that up to $50 \%$ of patients with concomitant hypertension and dyslipidaemia do not achieve their BP and lipid goals. ${ }^{(3,7,8)}$

A few studies have previously looked at the treatment patterns and factors associated with BP goal attainment in patients with concomitant hypertension and dyslipidaemia, but these studies were mainly retrospective in nature and none were conducted in Asian populations. ${ }^{(1,2,7,8)}$ Singapore has a multi-ethnic Asian population. Coronary arterial diseases and strokes are the top two major causes of deaths globally. The World Health Organization reported that the local population in Singapore had the fourth longest life span in 2014. ${ }^{(9)}$ The National Health Survey 2010 by the Ministry of Health, Singapore, showed that among adults aged $30-69$ years, the prevalence of hypertension was $23.5 \%$, while those of high total cholesterol levels (> $6.2 \mathrm{mmol} / \mathrm{L})$ and high LDL-C levels (> $4.1 \mathrm{mmol} / \mathrm{L}$ ) were $17.4 \%$ and $15.2 \%$, respectively. The survey also found that $49.5 \%$ of all known patients with

${ }^{1}$ SingHealth Polyclinics - Pasir Ris, ${ }^{2}$ Duke-NUS Medical School, ${ }^{3}$ SingHealth Polyclinics - Pharmacy, ${ }^{4}$ SingHealth Polyclinics - Tampines, ${ }^{5}$ SingHealth Polyclinics - Research, Singapore Correspondence: Dr Koh Kim Hwee, Consultant, SingHealth Polyclinics - Pasir Ris, 1 Pasir Ris Drive 4, \#01-11, Singapore 519457. koh.kim.hwee@singhealth.com.sg 
hypertension had good BP control, while the proportion among those on treatment was $52.9 \%$. $^{(10)}$

In the 2010 Primary Care Survey in Singapore, hypertension and dyslipidaemia were the top medical conditions seen in all public primary care clinics. ${ }^{(11)}$ Therefore, the present study was undertaken primarily to assess the factors associated with BP goal attainment in patients with concomitant hypertension and dyslipidaemia attending two primary care clinics in Singapore. The secondary objective was to analyse the prescription patterns in association with treatment goals in this group of patients.

\section{METHODS}

This paper is a sub-analysis of the Lipid HEALTH study, which aimed to determine the effect of ethnic variation on the attainment of LDL-C treatment goals among multi-ethnic Asian patients with dyslipidaemia in primary care. The study design and methods of the Lipid HEALTH study, as well as the baseline results, have been previously reported in detail.(12)

Primary healthcare services in Singapore are delivered by both public polyclinics and private general practitioner clinics. Due to the provision of healthcare subsidies and privileges, $45 \%$ of the local Asian population consult polyclinics for the management of their chronic diseases, including dyslipidaemia and hypertension. ${ }^{(11)}$ The study was carried out in two typical, adjacent polyclinics in the Tampines and Pasir Ris housing estates in north-eastern Singapore, where the investigators are practising as primary healthcare professionals. Both polyclinics served about 258,810 multi-ethnic Asian residents (as of June 2014) who were sociodemographically similar to the rest of the Singapore population.

The Lipid HEALTH study ${ }^{(12)}$ targeted adult Asian patients aged 31-80 years with physician-diagnosed dyslipidaemia. Patients with cognitive, hearing and/or visual impairment who were incapable of providing written informed consent were excluded. This study took place from October 2013 to September 2014. Two trained research assistants (RAs) were assigned to screen patients for eligibility to be enrolled in the study, one at each study site. They approached potential patients in the consultation waiting areas and enquired if their medical conditions included lipid disorders; this was then verified against their electronic health records. Once the eligibility criteria were fulfilled, the RA explained the study using a participant information sheet (approved by the institutional review board) and clarified doubts and queries before obtaining the patient's written informed consent. Participation in the study was strictly voluntary. This recruitment method was carried out in a consecutive case-encounter fashion. The questionnaire was interviewer administered by the RA. In total, 851 patients with dyslipidaemia were found to have concurrent hypertension and included in the study. Standardised English-based questionnaires were used to collect information on patients' demographic characteristics and clinical information such as comorbidities. Laboratory data and prescription patterns, including the number and types of BP-lowering medications, retrieved from the electronic health records, were evaluated as independent variables.
Under a conservative assumption that the proportion of those who achieved LDL-C treatment goals was 50\%, the sample size required was 380 , based on a $5 \%$ level of significance and $95 \%$ confidence interval (Cl). To account for the smaller ethnic groups and to adequately power the study to answer the primary research question, the sample size was tripled (for Chinese, Malay and Indian ethnicities) to $1,140 .^{(12)}$

We used the JNC 7 guidelines ${ }^{(4)}$ to define our BP goals, which were the major outcomes of this study. BP goals were defined as either average systolic $\mathrm{BP} \leq 140 \mathrm{mmHg}$ and average diastolic $\mathrm{BP} \leq 90 \mathrm{mmHg}$ for all patients, excluding patients with diabetes mellitus (DM) or chronic kidney disease, or average systolic $\mathrm{BP} \leq 130 \mathrm{mmHg}$ and average diastolic $\mathrm{BP} \leq 80 \mathrm{mmHg}$ for patients with DM or chronic kidney disease.

The Framingham CHD risk score ${ }^{(13)}$ was used to categorise the patients into three risk groups that are susceptible to developing vascular diseases - high risk: patients with dyslipidaemia and any existing vascular diseases (Type 2 DM, ischaemic heart disease, stroke, renal disease, peripheral vascular disease); medium/ intermediate risk: patients with dyslipidaemia and hypertension alone; and low risk: patients with dyslipidaemia without other cardiovascular comorbidities.

Based on the Ministry of Health Singapore's 2006 clinical practice guidelines for the management of lipid disorders, LDL-C treatment goals were defined based on the cardiovascular risk profile. The recommended LDL-C goal levels were as follows - high-risk group: < $100 \mathrm{mg} / \mathrm{dL}$ (2.6 mmol/L); intermediaterisk group: < $130 \mathrm{mg} / \mathrm{dL}(3.4 \mathrm{mmol} / \mathrm{L})$; and low-risk group: $<160 \mathrm{mg} / \mathrm{dL}$ (4.1 mmol/L). The recommended triglyceride (TG) goal level for all patients was $<200 \mathrm{mg} / \mathrm{dL}(2.3 \mathrm{mmol} / \mathrm{L})$, and the high-density lipoprotein cholesterol (HDL-C) goal level for all patients was $\geq 40 \mathrm{mg} / \mathrm{dL}(1.0 \mathrm{mmol} / \mathrm{L}){ }^{\left({ }^{(14}\right.}$

Descriptive statistics were used to present the demographic characteristics of the study population, comparing patients who achieved their BP goals with those who did not. Differences between these groups were tested using chi-square test or Fisher's exact test for categorical variables, and independent $t$-test for continuous variables. All significant factors on demographics and medication were then entered into a logistic regression model to identify the adjusted odds ratio (OR) of factors associated with BP control. All analyses were performed using IBM SPSS Statistics version 22.0 (IBM Corp, Armonk, NY, USA). A p-value $<0.05$ was considered statistically significant. The study received approval from the SingHealth Centralised Institutional Review Board (CIRB reference 2013/630/E).

\section{RESULTS}

Based on the inclusion and exclusion criteria, a total of 851 patients with concomitant hypertension and dyslipidaemia were included in the sub-analysis. Table I shows the demographic characteristics of the patients who achieved their BP treatment goals and those who failed to reach their goals. Out of 851 patients with concomitant hypertension and dyslipidaemia, only $49.7 \%$ ( $n=423$ ) attained their BP goals. Gender was not associated with achievement of BP treatment goals. A greater proportion 
Table I. Characteristics of patients with hypertension and dyslipidaemia: demographics, use of antihypertensive medications and lipid profile in association with BP treatment goals.

\begin{tabular}{|c|c|c|c|c|}
\hline \multirow[t]{2}{*}{ Characteristic } & \multicolumn{3}{|c|}{ No. (\%) } & \multirow[t]{2}{*}{ p-value } \\
\hline & $\begin{array}{l}\text { Total } \\
(n=851)\end{array}$ & $\begin{array}{l}\text { BP goals not achieved } \\
(n=428)\end{array}$ & $\begin{array}{l}\text { BP goals achieved } \\
(n=423)\end{array}$ & \\
\hline \multicolumn{5}{|l|}{ Demographics } \\
\hline Gender & & & & 0.83 \\
\hline Female & $510(59.9)$ & $255(50.0)$ & $255(50.0)$ & \\
\hline Male & $341(40.1)$ & $173(50.7)$ & $168(49.3)$ & \\
\hline Ethnicity & & & & 0.06 \\
\hline Chinese & $270(31.7)$ & $120(44.4)$ & $150(55.6)$ & \\
\hline Malay & $297(34.9)$ & $161(54.2)$ & $136(45.8)$ & \\
\hline Indian & $284(33.4)$ & $147(51.8)$ & $137(48.2)$ & \\
\hline Age (yr) & & & & 0.58 \\
\hline$<60$ & $340(40.0)$ & $167(49.1)$ & $173(50.9)$ & \\
\hline$\geq 60$ & $511(60.0)$ & $261(51.1)$ & $250(48.9)$ & \\
\hline Education & & & & 0.35 \\
\hline Secondary or below & $737(86.6)$ & $366(49.7)$ & $371(50.3)$ & \\
\hline Diploma/tertiary & $114(13.4)$ & $62(54.4)$ & $52(45.6)$ & \\
\hline Employment status & & & & 0.57 \\
\hline Employed & $358(42.1)$ & $176(49.2)$ & $182(50.8)$ & \\
\hline Unemployed & $493(57.9)$ & $252(51.1)$ & $241(48.9)$ & \\
\hline Body mass index $\left(\mathrm{kg} / \mathrm{m}^{2}\right)$ & & & & $0.04^{+}$ \\
\hline$<23$ & $120(14.1)$ & $50(41.7)$ & $70(58.3)$ & \\
\hline$\geq 23$ & $729(85.9)^{*}$ & $377(51.7)$ & $352(48.3)$ & \\
\hline \multicolumn{5}{|l|}{ Lifestyle habit } \\
\hline Current smoker & & & & 0.52 \\
\hline Yes & $48(5.6)$ & $22(45.8)$ & $26(54.2)$ & \\
\hline No & $803(94.4)$ & $406(50.6)$ & $397(49.4)$ & \\
\hline Alcohol use & & & & 0.20 \\
\hline Yes & $15(1.8)$ & $10(66.7)$ & $5(33.3)$ & \\
\hline No & $836(98.2)$ & $418(50.0)$ & $418(50.0)$ & \\
\hline \multicolumn{5}{|l|}{ Comorbidity } \\
\hline Diabetes mellitus & & & & $<0.01^{+}$ \\
\hline Yes & $622(73.1)$ & $354(56.9)$ & $268(43.1)$ & \\
\hline No & $229(26.9)$ & $74(32.3)$ & $155(67.7)$ & \\
\hline Ischaemic heart disease/CAD & & & & $0.03^{+}$ \\
\hline Yes & $204(24.0)$ & $116(56.9)$ & $88(43.1)$ & \\
\hline No & $647(76.0)$ & $312(48.2)$ & $335(51.8)$ & \\
\hline Cerebral vascular disease/stroke/ TIA & & & & 0.43 \\
\hline Yes & $39(4.6)$ & $22(56.4)$ & $17(43.6)$ & \\
\hline No & $812(95.4)$ & $406(50.0)$ & $406(50.0)$ & \\
\hline Renal disease & & & & $<0.01^{+}$ \\
\hline Yes & $76(8.9)$ & $51(67.1)$ & $25(32.9)$ & \\
\hline No & $775(91.1)$ & $377(48.6)$ & $398(51.4)$ & \\
\hline Peripheral vascular disease & & & & 0.10 \\
\hline Yes & $6(0.7)$ & $5(83.3)$ & $1(16.7)$ & \\
\hline No & $845(99.3)$ & $423(50.1)$ & $422(49.9)$ & \\
\hline \multicolumn{5}{|l|}{ Medication } \\
\hline No. of hypertension medications & & & & $<0.01^{+}$ \\
\hline 0 & $46(5.4)$ & $16(34.8)$ & $30(65.2)$ & \\
\hline 1 & $315(37.0)$ & $133(42.2)$ & $182(57.8)$ & \\
\hline 2 & $276(32.4)$ & $150(54.3)$ & $126(45.7)$ & \\
\hline$\geq 3$ & $214(25.1)$ & $129(60.3)$ & 85 (39.7) & \\
\hline
\end{tabular}


Table I. (Contd...)

\begin{tabular}{|c|c|c|c|c|}
\hline \multirow[t]{2}{*}{ Characteristic } & \multicolumn{3}{|c|}{ No. (\%) } & \multirow[t]{2}{*}{ p-value } \\
\hline & $\begin{array}{l}\text { Total } \\
(n=851)\end{array}$ & $\begin{array}{l}\text { BP goals not achieved } \\
(n=428)\end{array}$ & $\begin{array}{l}\text { BP goals achieved } \\
(n=423)\end{array}$ & \\
\hline \multicolumn{5}{|l|}{ Lipid profile goal } \\
\hline LDL-C ${ }^{b}$ & & & & $<0.01^{\dagger}$ \\
\hline Not achieved & $219(25.7)$ & $141(64.4)$ & 78 (35.6) & \\
\hline Achieved & $632(74.3)$ & $287(45.4)$ & $345(54.6)$ & \\
\hline $\mathrm{HDL}-\mathrm{C}^{\mathrm{c}}$ & & & & 0.46 \\
\hline Not achieved & $116(13.6)$ & $62(53.4)$ & $54(46.6)$ & \\
\hline Achieved & $735(86.4)$ & $366(49.8)$ & $369(50.2)$ & \\
\hline Triglyceride $^{d}$ & & & & 0.13 \\
\hline Not achieved & $88(10.3)$ & $51(58.0)$ & $37(42.0)$ & \\
\hline Achieved & $763(89.7)$ & 377 (49.4) & $386(50.6)$ & \\
\hline
\end{tabular}

*The percentage was calculated based on 849 due to incomplete data for 2 patients. $\mathrm{tp}<0.05$ is considered statistically significant. ${ }^{\mathrm{BBP}}$ goals achieved: $<140 / 90 \mathrm{mmHg}$ for all patients (except patients with DM or renal disease) and $<130 / 80 \mathrm{mmHg}$ for patients with DM or renal disease; BP goals not achieved: $\geq 140 / 90 \mathrm{mmHg}$ for all patients (except patients with DM or renal disease) and $\geq 130 / 80 \mathrm{mmHg}$ for patients with DM or renal disease. 'bDL-C goals achieved: $\leq 160 \mathrm{mg} / \mathrm{dL}(4.1 \mathrm{mmol} / \mathrm{L})$ for low-risk group, $>130 \mathrm{mg} / \mathrm{dL}$ ( $3.4 \mathrm{mmol} / \mathrm{L})$ for medium-risk group, $\leq 100 \mathrm{mg} / \mathrm{dL}$ ( $2.6 \mathrm{mmol} / \mathrm{L})$ for high-risk group; LDL-C goals not achieved: $>160 \mathrm{mg} / \mathrm{dL}$ (4.1 mmol/L) for low-risk group, $>130 \mathrm{mg} / \mathrm{dL}$ ( $3.4 \mathrm{mmol} / \mathrm{L})$ for medium-risk group and $>100 \mathrm{mg} / \mathrm{dL}(2.6 \mathrm{mmol} / \mathrm{L})$ for high-risk group. cHDL-C goals achieved: $\geq 40 \mathrm{mg} / \mathrm{dL}$ ( $1.0 \mathrm{mmol} / \mathrm{L})$; HDL-C goals not achieved: $<40 \mathrm{mg} / \mathrm{dL}(1.0 \mathrm{mmol} / \mathrm{L})$. $\mathrm{d}$ Triglyceride goals achieved: $<200 \mathrm{mg} / \mathrm{dL}$ ( $2.3 \mathrm{mmol} / \mathrm{L})$; triglyceride goals not achieved: $\geq 200 \mathrm{mg} / \mathrm{dL}$ ( $(2.3 \mathrm{mmol} / \mathrm{L})$. BP: blood pressure; CAD: coronary artery disease; HDL-C: high-density lipoprotein cholesterol; LDL-C: low-density lipoprotein cholesterol; TIA: transient ischaemic attack

Table II. Types of medication and frequency of achievement of blood pressure (BP) treatment goals.

\begin{tabular}{|c|c|c|c|}
\hline \multirow[t]{2}{*}{ Group of hypertension medication* } & \multicolumn{3}{|c|}{ No. (\%) } \\
\hline & Total $(n=851)$ & BP goals not achieved ${ }^{+}(n=428)$ & BP goals achieved ${ }^{\ddagger}(n=423)$ \\
\hline Beta-blocker & $329(38.7)$ & $175(53.2)$ & $154(46.8)$ \\
\hline Calcium channel blocker & $442(51.9)$ & $235(53.2)$ & $207(46.8)$ \\
\hline Angiotensin-converting enzyme inhibitor & $261(30.7)$ & $147(56.3)$ & $114(43.7)$ \\
\hline Angiotensin II receptor blocker & $331(38.9)$ & $180(54.4)$ & $151(45.6)$ \\
\hline Diuretics & $192(22.6)$ & $112(58.3)$ & $80(41.7)$ \\
\hline Other BP-lowering medications ${ }^{\varsigma}$ & $12(1.4)$ & $10(83.3)$ & $2(16.7)$ \\
\hline
\end{tabular}

*Patients can take medications from more than one group. †BP goals not achieved: $\geq 140 / 90 \mathrm{mmHg}$ for all patients (except patients with DM or renal disease) and $\geq 130 / 80 \mathrm{mmHg}$ for patients with DM or renal disease. $\neq B P$ goals achieved: $<140 / 90 \mathrm{mmHg}$ for all patients (except patients with DM or renal disease) and $<130 / 80$ $\mathrm{mmHg}$ for patients with DM or renal disease. §Patients can take medications from more than one group; other antihypertensive medications include methyldopa, hydralazine, prazosin and terazosin. DM: diabetes mellitus

of Chinese patients achieved their BP goal (55.6\%) compared to their Malay (45.8\%) and Indian (48.2\%) counterparts, but this was not statistically significant. Significantly more patients who were overweight (BMI $\geq 23 \mathrm{~kg} / \mathrm{m}^{2}$ ) failed to achieve treatment goals (51.7\%). DM, ischaemic heart disease/coronary artery disease and renal disease were associated with greater proportions of patients not achieving BP goals.

Most patients with concomitant hypertension and dyslipidaemia (94.6\%) were prescribed at least one BP-lowering medication: $37.0 \%$ were on monotherapy and more than half (57.6\%) were on two or more BP-lowering medications. 5.4\% of patients were on conservative management with diet alone. None of the patients were on single-pill combined medications. Among those on pharmacotherapy ( $\mathrm{n}=805), 51.2 \%$ failed to achieve their BP goals. Those who were not on any BP-lowering medication or on monotherapy were more likely to achieve their BP goals compared to those on two or more medications $(p<0.01)$. The average number of BP-lowering medication prescribed was 1.9 .
All the patients studied were of at least medium cardiovascular risk, given that they all had concomitant hypertension and dyslipidaemia. Those who achieved their LDL-C treatment goals were also more likely to achieve their BP treatment goals $(p<0.01)$. However, achieving HDL-C and triglyceride treatment goals were not associated with attainment of BP treatment goals.

Table II shows the types of BP-lowering medications prescribed and the frequency of achievement of BP treatment goals. Calcium channel blockers (CCBs) were the most frequently used BP-lowering agent $(51.9 \%, \mathrm{n}=442)$, followed by angiotensin II receptor blockers (ARBs; 38.9\%, $\mathrm{n}=331$ ), beta-blockers $(38.7 \%, \mathrm{n}=329)$, angiotensin-converting enzyme inhibitors (ACEls; 30.7\%, $\mathrm{n}=261$ ) and diuretics (22.6\%, $\mathrm{n}=192)$. The types of BP-lowering medications were comparable between patients who achieved their BP goal and those who did not.

Among the beta-blockers, atenolol was the most commonly used $(78.1 \%)$, followed by bisoprolol (21.6\%). Amlodipine was the most commonly prescribed CCB $(61.8 \%)$, followed by nifedipine $(35.5 \%)$. For the ACEls, the most commonly 
Table III. Factors influencing attainment of BP goal in patients with hypertension and dyslipidaemia using logistic regression analysis.

\begin{tabular}{|lll|}
\hline Factor & OR $(\mathbf{9 5} \% \mathbf{C l})$ & p-value \\
\hline \begin{tabular}{lll} 
Body mass index $\left(\mathbf{k g} / \mathbf{m}^{2}\right)$ \\
\hline 23
\end{tabular} & 1 & - \\
\hline$\geq 23$ & $1.18(0.78-1.78)$ & 0.44 \\
\hline Diabetes mellitus & 1 & - \\
\hline Yes & $2.27(1.61-3.13)$ & $<0.01^{\ddagger}$ \\
\hline No & & \\
\hline Ischaemic heart disease/CAD & 1 & - \\
\hline Yes & $1.25(0.90-1.75)$ & 0.18 \\
\hline No & & \\
\hline Renal disease & 1 & - \\
\hline Yes & $1.67(0.99-2.80)$ & 0.05 \\
\hline No & & \\
\hline No. of hypertension medications & & - \\
\hline$\geq 3$ & 1 & $0.03^{\ddagger}$ \\
\hline 0 & $2.19(1.09-4.39)$ & $<0.01^{\ddagger}$ \\
\hline 1 & $1.71(1.18-2.48)$ & \\
\hline 2 & $1.13(0.77-1.64)$ & 0.53 \\
\hline LDL-C & & - \\
\hline Goals not achieved* & 1 & $<0.01^{\ddagger}$ \\
\hline Goals achieved & & \\
\hline
\end{tabular}

*LDL-C goals not achieved: LDL > $160 \mathrm{mg} / \mathrm{dL}(4.1 \mathrm{mmol} / \mathrm{L})$ for low-risk group, LDL > $130 \mathrm{mg} / \mathrm{dL}(3.4 \mathrm{mmol} / \mathrm{L})$ for medium-risk group and LDL $>100 \mathrm{mg} / \mathrm{dL}(2.6 \mathrm{mmol} / \mathrm{L})$ for high-risk group. $+\mathrm{LDL}-\mathrm{C}$ goals achieved: LDL $\leq 160 \mathrm{mg} / \mathrm{dL}(4.1 \mathrm{mmol} / \mathrm{L})$ for low-risk group, $\mathrm{LDL}>130 \mathrm{mg} / \mathrm{dL}(3.4 \mathrm{mmol} / \mathrm{L})$ for medium-risk group and $\mathrm{LDL} \leq 100 \mathrm{mg} / \mathrm{dL}(2.6 \mathrm{mmol} / \mathrm{L})$ for high-risk group. $\neq \mathrm{p}<0.05$ is considered statistically significant. BP: blood pressure; CAD: coronary artery disease; $\mathrm{Cl}$ : confidence interval; LDL-C: low-density lipoprotein cholesterol; OR: odds ratio

prescribed was enalapril (61.3\%), followed by lisinopril (31.0\%) and perindopril (6.1\%). For the ARBs, losartan was prescribed in $73.3 \%$ of cases, telmisartan in $17.3 \%$ and valsartan in $8.5 \%$. Hydrochlorothiazide was the most commonly prescribed diuretic (89.1\%).

Logistic regression analysis (Table III) showed that attainment of the BP treatment goal was significantly more likely in those without Type 2 DM (OR 2.27, 95\% Cl 1.61-3.13), those who were able to achieve LDL-C goals (OR 2.02, 95\% Cl 1.45-2.81), and those who were not on any BP-lowering medication (OR $2.19,95 \% \mathrm{Cl} 1.09-4.39$ ) or on BP-lowering monotherapy (OR $1.71,95 \% \mathrm{Cl} 1.18-2.48)$.

\section{DISCUSSION}

Our data showed that only $49.7 \%$ of the patients in our local population who attended primary care achieved their BP goals. Even among those who were taking BP-lowering medications, $51.2 \%$ failed to achieve their targeted BP levels. This was consistent with several previous studies conducted on patients with concomitant hypertension and dyslipidaemia, which reported BP goal attainment rates of $15 \%-54 \%$. $^{(1-3,15,16)}$ Studies conducted in patients with isolated hypertension or dyslipidaemia have also shown that among patients who were treated, less than half reached their relevant therapeutic goal. ${ }^{(8)}$
In patients with hypertension, it is critical to control not only BP but also other cardiovascular risk factors. This low rate of BP goal attainment is particularly alarming, as concomitant hypertension and dyslipidaemia is prevalent in the local population. Several factors may contribute to poor BP goal attainment, and these include inadequate therapy (insufficient number of medications or sub-therapeutic doses), poor adherence to medications or lifestyle modifications, therapeutic inertia, and any combination of these barriers. ${ }^{(4,8,16)}$

For effective control of $\mathrm{BP}, \mathrm{JNC} 7^{(4)}$ recommends a combination of two or more lifestyle modifications, such as the Dietary Approaches to Stop Hypertension eating plan, dietary sodium reduction, physical activity, and weight reduction in overweight or obese patients, in addition to BP-lowering medications. It has also been suggested that more than half to two-thirds of hypertensive patients will require two or more BP-lowering medications to achieve their BP goal. ${ }^{(4,17)}$ Chopra and Kamal found that patients who were prescribed one or more antihypertensive medications were less likely to attain BP goals and dual BP/LDL-C goals. ${ }^{(2)}$

Multiple medications may be required as the disease progresses with time, but such prescriptions may impact patients' medication adherence. Lack of compliance with these behavioural modifications, coupled with a complex, multiple-medication regimen, might result in a lower likelihood of attaining BP goals. For example, it has been shown that only $36 \%$ of patients were adherent to both BP- and lipid-lowering medications after one year of treatment, which can further explain the lack of goal attainment in most patients. ${ }^{(18)}$ In this study, we found that patients prescribed two or more BP-lowering medications were less likely to attain BP goals. Furthermore, the likelihood of attaining BP goals decreased with an increasing number of BP-lowering medications prescribed. Conversely, those who were not on any BP-lowering medication (i.e. on lifestyle modifications alone) were likely to already have better BP control and, thus, a higher proportion of patients in this category attained their BP goals compared to those who were on pharmacotherapy.

BP-lowering medications can be prescribed in various combinations to achieve BP goals, and the average reduction in BP level with each of the classes of medications is comparable at the recommended dosages. ${ }^{(17)}$ The selection of BP-lowering medication is based on compelling indications such as the presence of chronic renal disease, DM, heart failure, postmyocardial infarction, angina pectoris and arrhythmias. ${ }^{(17)}$ Other factors include contraindications, physician experience and patient acceptance. In this study, CCBs were the most commonly prescribed class of BP-lowering medications, followed by ARBs, beta-blockers, ACEls and diuretics. The high frequency of CCB prescriptions could be related to their listing in the institution drug formulary over a long period of time. As noted in JNC 7, diuretics remain underutilised despite their various benefits. ${ }^{(4)}$

Current guidelines support the combined use of more than one class of medications to achieve BP goals. ${ }^{(4,19)}$ This strategy may increase the likelihood of achieving the target BP in a shorter time period, requiring fewer clinic visits and medication changes. 
However, pill burden is associated with lower adherence. ${ }^{(20)}$ The use of single-pill, fixed-dose combination preparations may reduce pill burden, improve patient compliance and BP control and, if both drugs are given at lower doses, reduce side effects. ${ }^{(19,20)}$ JNC 7 also suggested that the use of fixed-dose combinations may be more convenient and may also simplify the treatment regimen. ${ }^{(4)}$ However, fixed-dose combinations can be expensive for patients in fee-for-service healthcare systems, including that of Singapore, which may in turn constitute a barrier to adherence. No patient in this study was prescribed with single-pill, fixeddose combined medications. Aside from cost, these combined medications are not on the list of subsidised drugs, which could have influenced the physicians' prescribing behaviour.

Hypertension and dyslipidaemia frequently coexist in the same individual, and patients often require treatment for both conditions concomitantly. Many landmark BP therapeutic and statin trials included a large proportion of patients with concomitant hypertension and dyslipidaemia. ${ }^{(21)}$ In the Heart Protection Study, substantial reductions in total cholesterol levels and CHD events were observed in statin-treated patients, $41 \%$ of whom had a history of hypertension. ${ }^{(22)}$ In our study, attainment of BP treatment goals was significantly associated with the concurrent attainment of LDL-C goals. Barrios et al also found an association between BP and LDL-C goal attainment in a similar study in Spain. ${ }^{(16)}$ This association may be because patients who are adherent to medications and lifestyle modifications for dyslipidaemia are also more likely to be adherent to their hypertension treatment. Bundling the treatment together (i.e. the combined use of BP-lowering medication and statin) could be a more efficient approach to attaining goals and improving medication adherence, but such combined medications require safety and effectiveness evaluations, as well as further research.

The presence of comorbidities often leads to polypharmacy and increased pill burden, adding more challenges to such complex disease management. ${ }^{(20)}$ The presence of DM is a major predictor of suboptimal BP control. ${ }^{(1-3,16)}$ Based on JNC 7 guidelines, the BP goal is lower for patients with DM or renal impairment as compared with those without these conditions (<130/80 mmHg vs. < 140/90 mmHg). ${ }^{(4)}$ Thus, diabetic patients are likely to be on more medications to achieve the lower BP goals, which may conversely increase their likelihood of poorer medication adherence.

The low proportion of patients whose BP was treated to goal highlights the need for further improvement in this area. Numerous institutional measures have been undertaken to address these barriers via system approaches, quality improvement initiatives and research. BP goal attainment is a quality indicator that is captured monthly and monitored across all SingHealth Polyclinics. ${ }^{(23)}$ Quality improvement projects have since been undertaken in each polyclinic to identify the gaps and increase the proportion of patients achieving their treatment goals. Successful interventions are also shared across the polyclinics at quarterly inter-polyclinic collaborative meetings.

Failure to titrate/combine medications and reinforce lifestyle modifications despite knowing that the patient is not achieving BP goals represents clinical inertia, which must be overcome. This may be due, in part, to clinicians' focus on relieving symptoms, lack of familiarity with clinical guidelines or discomfort with titrating to a goal. ${ }^{(4)}$ All new doctors posted to SingHealth Polyclinics undergo intensive induction, including the management of hypertension. A doctor's guidebook, which is regularly updated, also serves to assist physicians in managing hypertension according to evidence-based guidelines. ${ }^{(24)}$ Several combination drugs have since been introduced into the institution formulary, even though they have yet to be on the list of approved subsidised medications. This allows the physicians to have greater flexibility in their choice of BP-lowering medications, especially when targeting patients with poor medication adherence.

Research endeavours also contribute to the effort to expand the hypertensive population in the entire institution who achieve treatment goals. After the successful completion of a feasibility study, ${ }^{(25)}$ a multi-centre open randomised controlled trial was launched across eight polyclinics in the institution to determine the effectiveness of protocolised management of hypertension and its risk factors as well as the cost-effectiveness of singlepill, fixed-dose combined BP-lowering medication. One of the potential outcomes of this trial is an increase in the proportion of patients who can attain treatment goals for both hypertension and dyslipidaemia at the intervention sites.

For the current study, the investigators used a simplified risk stratification framework in the institution, which was modified from the Framingham risk scores, to reflect real-time clinical practice during the period of the survey. The simplified version was used for ease of implementation in a high-volume public primary healthcare system, where each polyclinic manages between 500 to 1,100 patients per day. This study, however, was not without limitations. The study population was recruited from the north-eastern district of Singapore with disproportionate representation of the local ethnic groups. Hence, caution should be exercised before extrapolating the results to the local general population. Furthermore, the self-reported method of data collection could be subjected to recall bias, although such an approach is widely used in routine clinical practice in view of time and resource constraints. This self-reported data from patients without objective verification may also limit the generalisability of our results.

Overall, about half of our study population with concomitant hypertension and dyslipidaemia failed to attain their BP treatment goals, despite established disease management guidelines. The presence of DM and suboptimal dyslipidaemia control were associated with suboptimal BP control. Managing patients with multiple cardiovascular risk factors such as hypertension and dyslipidaemia should involve simultaneous management and attainment of their BP and lipid goals.

\section{ACKNOWLEDGEMENTS}

The authors are grateful to the Singapore Heart Foundation for the kind sponsorship of this study (grant number: RG2012/07). We would also like to thank our research assistants, Ms Ng Chiat Eng and Ms Xu Hui Yan, for their diligence in completing the 
subject recruitment within the time period of the study, and team members, nurse Tan Xin Yi and Dr Chan Hian Hui Vincent, for their assistance.

\section{REFERENCES}

1. Egan BM, Li J, Qanungo S, Wolfman TE. Blood pressure and cholesterol contro in hypertensive hypercholesterolemic patients: national health and nutrition examination surveys 1988-2010. Circulation 2013; 128:29-41.

2. Chopra I, Kamal KM. Factors associated with therapeutic goal attainment in patients with concomitant hypertension and dyslipidemia. Hosp Pract (1995) 2014; 42:77-88

3. Welch V, Tang SS. Treatment and control of BP and lipids in patients with hypertension and additional risk factors. Am J Cardiovasc Drugs 2007; 7:381-9.

4. Chobanian AV, Bakris, GL, Black HR, et al. The seventh report of the Join National Committee on prevention, detection, evaluation, and treatment of high blood pressure: the JNC 7 report. JAMA 2003; 289:2560-72.

5. European Society of Hypertension-European Society of Cardiology Guidelines Committee. 2003 European Society of Hypertension-European Society of Cardiology guidelines for the management of arterial hypertension. J Hypertens 2003; 21:1011-53.

6. Expert Panel on Detection, Evaluation, and Treatment of High Blood Cholesterol in Adults. Executive summary of the third report of the National Cholestero Education Program (NCEP) Expert Panel on Detection, Evaluation, and Treatment of High Blood Cholesterol in Adults (Adult Treatment Panel III). JAMA 2001; 285:2486-97.

7. Chopra I, Kamal KM, Candrilli SD, Kanyongo G. Association between obesity and therapeutic goal attainment in patients with concomitant hypertension and dyslipidemia. Postgrad Med 2014; 126:66-77.

8. Johnson ML, Pietz K, Battleman DS, Beyth RJ. Therapeutic goal attainment in patients with hypertension and dyslipidemia. Med Care 2006; 44:39-46.

9. World Health Organization. World health statistics 2014. Available at: http:/ apps.who.int/iris/bitstream/10665/112738/1/9789240692671_eng.pdf?ua=1 Accessed April 18, 2017.

10. Ministry of Health, Singapore. National Health Survey 2010. Available at: https://www.moh.gov.sg/content/moh_web/home/Publications/Reports/2011/ national_health_survey2010.html. Accessed March 28, 2017.

11. Sng QS. Primary care survey 2010. Profile of primary care patients. In: Ministry of Health, Singapore [online]. Available at: https://www.moh.gov.sg/content/ dam/moh_web/Publications/Information\%20Papers/2011/Primary\%20Care\%20 Survey\%202010\%20-\%20Profile\%20of\%20Primary\%20Care\%20Patients.pdf.
Accessed March 28, 2017.

12. Tan NC, Koh KH, Goh CC, Koh YL, Goh SC. Asian patients with dyslipidemia in an urban population: effect of ethnicity on their LDL-cholesterol treatment goals. J Clin Lipidol 2016; 10:410-9.

13. Wilson PW, D'Agostino RB, Levy D, et al. Prediction of coronary heart disease using risk factor categories. Circulation 1998; 97:1837-47.

14. Ministry of Health, Singapore. Clinical practice guidelines - lipids (2006). Available at: https://www.moh.gov.sg/content/dam/moh_web/HPP/Doctors/ cpg_medical/withdrawn/cpg_Lipids-May\%202006.pdf. Accessed March 28, 2017. Withdrawn in: https://www.moh.gov.sg/docs/librariesprovider4/ guidelines/cpg_lipids-may-2006.pdf.

15. Ohta Y, Tsuchihashi T, Morinaga Y, Onaka U, Ueno M. Blood pressure and lipid control status in Japanese hypertensive patients. Clin Exp Hypertens 2009; 31:298-305.

16. Barrios V, Escobar C, Calderón A, et al. Blood pressure and lipid goal attainment in the hypertensive population in the primary care setting in Spain. J Clin Hypertens (Greenwich) 2007; 9:324-9.

17. Chobanian AV. Guidelines for the management of hypertension. Med Clin North Am 2017; 101:219-27.

18. Chapman RH, Benner JS, Petrilla AA, et al. Predictors of adherence with antihypertensive and lipid-lowering therapy. Arch Intern Med 2005; 165:1147-52.

19. Ferdinand KC, Nasser SA. Management of essential hypertension. Cardiol Clin 2017; 35:231-46.

20. Vrijens B, Antoniou S, Burnier M, de la Sierra A, Volpe M. Current situation of medication adherence in hypertension. Front Pharmacol 2017; 8:100

21. Nash DT. The clinical implications and management of concomitant hypertension and dyslipidemia. Postgrad Med 2006; 119:37-45.

22. Heart Protection Study Collaborative Group. MRC/BHF Heart Protection Study of cholesterol lowering with simvastatin in 20,536 high-risk individuals: a randomised placebo-controlled trial. Lancet 2002; 360:7-22.

23. Singhealth Polyclinics, Singapore. Clinical care performance 2009 to 2013. Available at: https://polyclinic.singhealth.com.sg/AboutUs/CorporateProfile/ Documents/ClinicalCarePerformance\%20Q3\%202013.pdf. Accessed April 18, 2017.

24. Ministry of Health, Singapore. Clinical practice guidelines - hypertension (2005). Available at: https://www.moh.gov.sg/content/dam/moh_web/HPP/Doctors/ cpg_medical/withdrawn/cpg_Hypertension-Jun\%202005.pdf. Accessed March 28, 2017.

25. Jafar TH, Tan NC, Allen JC, et al. Management of hypertension and multiple risk factors to enhance cardiovascular health - a feasibility study in Singapore polyclinics. BMC Health Serv Res 2016; 16:229 